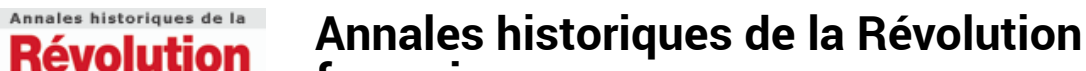

française française

326 | octobre- décembre 2001

La Révolution Batave. péripéties d'une République-

Soeur (1795-1813)

\section{La fête révolutionnaire aux Pays-Bas (1780-1806). De l'utopie à l'indifférence}

Frans Grijzenhout

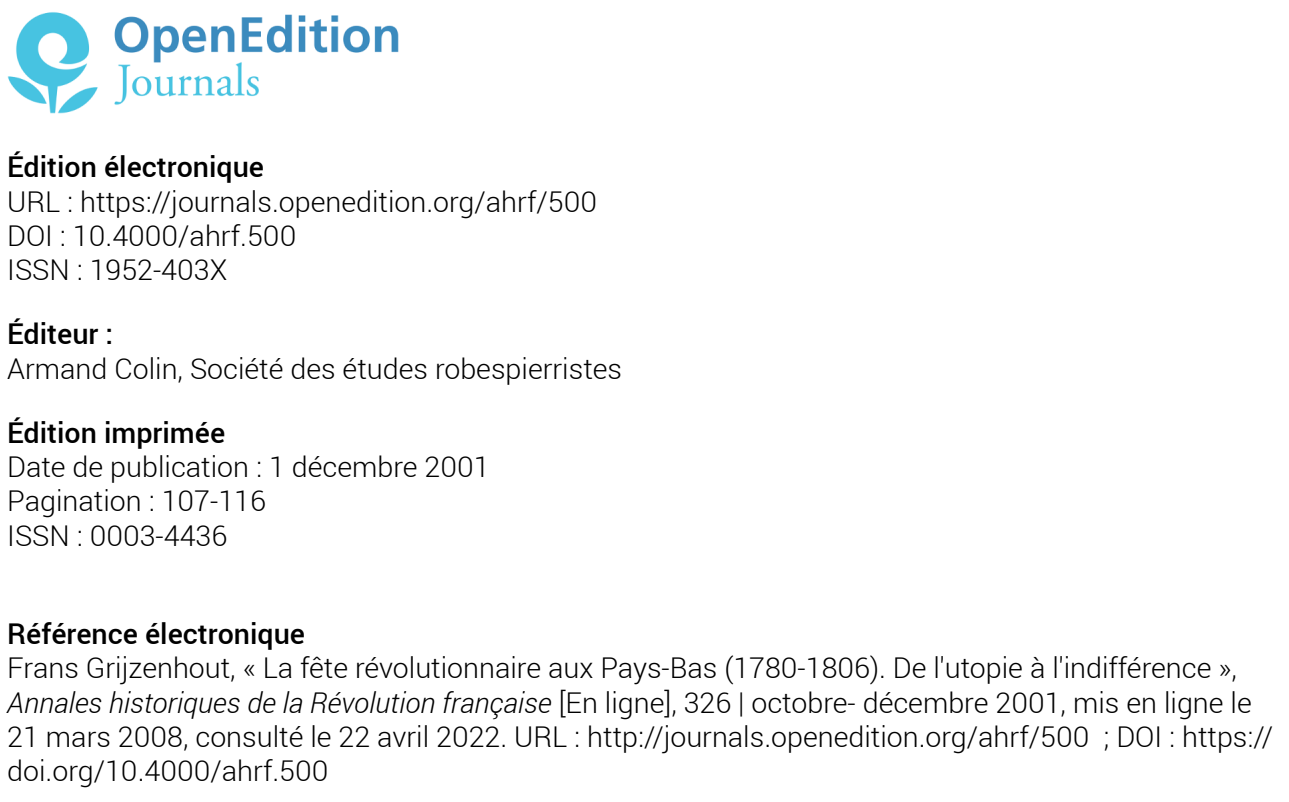

Tous droits réservés 


\title{
LA FÊTE RÉVOLUTIONNAIRE AUX PAYS-BAS (1780-1806) DE L'UTOPIE À L'INDIFFÉRENCE
}

\author{
FRANS GRIJZENHOUT
}

\begin{abstract}
Les fêtes patriotes célébrées entre 1781 et 1787 sont intéressantes à plus d'un titre. D'une part, elles empruntent au passé national et réactualisent une iconographie spécifique; d'autre part, elles renient tout ce qui a trait à la famille d'Orange. Leur caractère hautement utopique les sépare des fêtes françaises des années 1789-1794, mais aussi des fêtes bataves, plus inspirées du modèle parisien, qui ne parviendront pas à enthousiasmer la République-sœur, peu soucieuse en outre de gaspiller les fonds publics à cet usage. Mieux valait, pensait le ministre de l'Éducation nationale, réorganiser I'instruction publique.
\end{abstract}

Mots clés : fête révolutionnaire ; décors ; iconographie ; identité nationale ; utopie.

Depuis les travaux de Mona Ozouf, la fête révolutionnaire française nous est devenue plus compréhensible du point de vue de ses formes et de ses significations (1). Des recherches récentes ont révélé que le phénomène s'était étendu jusqu'en Italie, en Allemagne et dans d'autres pays européens (2). La République batave (1795-1806) elle aussi a suivi le mouvement et a connu une vogue modeste de fêtes révolutionnaires, au lendemain de la révolution batave de janvier 1795 (3). Mais, contrairement

(1) M. Ozouf, La fête révolutionnaire, 1789-1799, Paris, 1976. Voir aussi J. EHRARD \& P. VIALLANEIX (éd.), Les fêtes de la Révolution, Colloque de Clermont-Ferrand (juin 1974), Paris, 1977 et I. BAXMANN, Die Feste der Französischen Revolution. Inszenienung von Gesellschaft als Natur, Berlin, 1989.

(2) C. M. BOSSENO, «Iconographie des fêtes révolutionnaires italiennes (1796-1799), dans M. Vovelle (éd.), Les images de la Révolution française, Paris, 1988, pp. 157-164. O. DoTzEnROD, «Republikanische Feste in Rheinlandzur Zeit der Französische Revolution», dans D. DudiNG, P. Friedemann, P. MUNCh (éd.), Oeffentliche Festkultur. Politische Feste in Deutschland von der Aufklärung bis zum Ersten Weltkrieg, Reinbek bei Hamburg, 1988, pp. 46-66.

(3) F. GRIJZENHOUT, Feesten voor het Vaderland. Patriotse en Bataafse feesten, Amsterdam, 1989.

Annales historiques de la Révolution française - 2001 - N 4 [107 à 116] 
aux autres Républiques-sœurs, les Pays-Bas avaient déjà une tradition en ce domaine. Dans les années 1780-1787, au cours desquelles les patriotes avaient en vain tenté de saisir le pouvoir et de réformer le gouvernement de la République des Provinces-Unies, la fête avait été d'une grande importance dans les polémiques violentes qui opposaient orangistes et patriotes.

\section{La fête patriote}

Dans un numéro antérieur des A.H.R.F, un article a été consacré aux changements et évolutions subis par la fête patriote dans les années 1780-1787 (4). Au début, en effet, la fête avait un caractère essentiellement fermé. Elle consistait en un repas patriotique où participaient principalement des régents anti-orangistes. Et, à la fin du repas seulement, le public éventuel pouvait venir admirer les décorations symboliques ou allégoriques, placées tout autour de la table et destinées à exprimer les sentiments patriotiques des participants. De même, les textes des discours prononcés étaient publiés par la suite, accompagnés d'une description précise des décorations et du déroulement des festivités. La conclusion qui découlait de cette première enquête était que, sous cette forme, la fête patriote avait un caractère hautement utopique, fort différent de celui de la fête révolutionnaire française. Les patriotes se voyaient assis dans le «jardin hollandais », à l'ombre d'un arbre dont ils semaient les graines. À l'oranger, symbole traditionnel de la Maison d'Orange, qu'ils comparaient à l'arbre de la science du Bien et du Mal du Paradis, ils opposaient leur « arbre de la science politique et gouvernementale " (5).

L'image du jardin hollandais idéal fut représentée sous sa forme la plus poussée dans les décorations d'un banquet patriote, organisé le 24 avril 1786 à Rotterdam, à l'occasion de l'alliance conclue entre la France et les Provinces-Unies, le 10 novembre 1785 . La salle de fête tout entière figurait le jardin hollandaị, où se trouvaient à la place d'honneur les héros de la tradition anti-orangiste : Brederode, Oldenbarneveldt, Grotius, De Witt. Les gardiens du jardin étaient incarnés par les héros contemporains: Zoutman, Capellen, et lés Français Suffren et Bouillé. Cette fête rivalisait de splendeur et de luxe avec le banquet en l'honneur de l'alliance, célébré à Amsterdam le 27 janvier 17866. Là aussi, plusieurs décorations sur des thèmes patriotes, tels que le temple de la Liberté, le rétablissement des droits et des privilèges provinciaux, la liberté maritime, la prospérité de la

(4) F. Gruzenhout, «Le temple et la table, la fête patriote 1781-1787 ", A.H.R.F, n 277, 1989, pp. 185-196.

(5) Sur la symbolique naturelle de la Révolution française, H. C. et E. HARTEN, Die Versöhnung mit der Natur. Gärten, Freiheitsbäume, republikanische Wälder, heilige Bergen und Tugendsparks in der Französischen Revolution, Hamburg, 1989. 
France et de la République avaient orné la table pendant le dessert. En 1786 , et toujours en vue de chanter l'alliance, les fêtes se multiplièrent un peu partout en Hollande, sous forme de banquet privé ou bien sous forme d'illuminations publiques d'un édifice investi par une société patriote. Les décorations avaient toutes pour thème principal le motif de dextrarum iunctio, qui symbolisait l'alliance entre les deux pays. Certains éléments de décorations présentés lors de ces fêtes évoquaient les affinités entre cette alliance et l'alliance ancienne conclue autrefois entre les Bataves ou celle encore qu'avaient close les Gueux durant la révolte hollandaise du XVIe siècle contre l'Espagne.

Les patriotes n'organisaient pas seulement des festivités privées; au rythme de leurs succès, ils se manifestaient de plus en plus dans la rue. Virent alors le jour des rites publics pour les milices composées pour une grande part de membres de la moyenne et de la petite bourgeoisie. Le banquet patriote et les rites bourgeois trahissaient le processus de prise de conscience des patriotes, tel qu'il s'était élaboré depuis les années 17821783. Dans les théories qui se constituèrent peu à peu sur l'armement civil, où se décelait une influence quasi religieuse, divers modèles étaient perceptibles : celui de l'Amérique, de la Suisse, mais aussi des ancêtres hollandais du XVIe siècle. Ces modèles renvoyaient tous en fait à l'image idéale de la milice civile de l'ancienne Rome. Cette image de la République idéale, digne d'imitation et calquée sur l'exemple antique mais aussi sur les exemples batave, suisse ou américain allait devenir un thème important dans la rhétorique, le rituel et l'iconographie patriotes.

Si l'iconographie des banquets patriotes s'inscrivait surtout dans la tradition des régents républicains, celle des milices appartenait à une tradition exaltant l'autonomie urbaine et les vertus bourgeoises classiques. Les milices patriotes empruntaient les éléments de leur iconographie aux milices bourgeoises des XVI et XVII e siècles. À la différence près que désormais les allusions à la Maison d'Orange qui ornaient les bannières des anciens corps francs étaient devenues taboues. En leur lieu et place, les patriotes substituèrent les motifs de patrie et de liberté, qui prirent le devant de la scène dans l'iconographie des milices. Le motif de "liberté " référait tout d'abord à la restauration des anciens privilèges des villes et à la révolte des Hollandais contre l'Espagne. La représentation allégorique de la Liberté était tout à fait conforme à la tradition iconographique de la République, telle qu'elle avait été formulée en 1570. Dans les cercles des corps armés, le motif de Patrie était compris surtout comme local, parfois comme régional, et, de temps à autre, comme national.

Entre 1786 et $\mathbf{1 7 8 7}$, sommet du mouvement, l'installation de quelques nouveaux conseils municipaux accéléra l'évolution de la fête patriote: d'événement privé, se déroulant à l'intérieur, elle se fit événement public, se tenant à l'extérieur. Alors qu'à l'origine, les invités étaient voués à demeurer 
des spectateurs passifs, assis autour d'un temple en sucreries, posé sur une table, ils devinrent des participants à part entière dans une cérémonie, qui avait lieu dans un temple de la Liberté, érigé provisoirement au centre de la ville. Dans les cercles patriotes des années 1780 , le temple, tout comme le jardin, n'était plus le symbole d'une vertu (Liberté, Unité) mais une image en harmonie avec les aspirations patriotes et une utopie politique, symbolisée par une forme architecturale. Le patriotisme lui-même était considéré comme un temple de la Liberté en voie de construction (6). Ainsi, la fête patriote, plus encore peut-être que les fêtes de la Révolution française, voire de la révolution batave, exprimait une utopie et un monde idéal à venir (7).

L'iconographie patriote est explicitement néerlandaise. Y sont absentes les influences étrangères, y compris celle de l'Amérique insurgée. Elle se fonde pour une grande part sur l'iconographie politique de la République conçue au moment de la guerre de libération contre l'Espagne. En témoignent la réactualisation explicite des allégories de la Liberté, de la Patrie (le jardin hollandais), le serment des Gueux et plusieurs devises originaires de l'époque. Parallèlement, étaient modifiés les éléments qui ne concordaient pas avec les priorités politiques des patriotes. Les thèmes portant sur la religion et l'alliance avec la Maison d'Orange, privilégiés durant la Révolte, furent complètement occultés. Les patriotes par contre renouaient avec la tradition anti-orangiste et revalorisaient les vertus bourgeoises néerlandaises ou les vertus héroïques de l'antique Rome.

\section{L'exil}

À l'automne 1787, les troupes prussiennes mirent fin au rêve des patriotes et restaurèrent dans ses droits le Stadhouder. Avec lui revinrent les fêtes orangistes. En 1788, furent célébrés les anniversaires de Guillaume V et de son épouse, la princesse Wilhelmina de Prusse. Les patriotes n'avaient plus que l'espace privé pour organiser des banquets. Tel celui qui en 1794 célébra l'anniversaire d'un de leurs héros : le maire d'Amsterdam, Henrik Hooft. Entre-temps des milliers de patriotes avaient quitté la République, d'abord pour la Belgique, ensuite pour la France. Nombre d'entre eux s'installèrent à Saint-Omer, Dunkerque et Béthune. C'est là qu'ils assistèrent aux fêtes révolutionnaires organisées entre 1790 et 1794 (8). C'est ainsi qu'en

(6) Nous n'avons aucune preuve que la franc-maçonnerie ait influencé le parti patriote. Voir aussi dans ce numéro des $A . H . R F$. l'article d'Anton VAN DE SANDE.

(7) À ce sujet, B. BACZKO, "Lutopie et les fêtes", Tijdschrifi voor de Studie van de Verlichting, nos 3-4, 1973, pp. 20-62. M. OzoUf, L'école de la France. Essais sur la Révolution, l'utopie et l'enseignement, Paris, 1984.

(8) F. GRIJZEnhout, «Le purgatoire des patriotes", dans W. FriJhofF \& R. DEKKER(éd.), Le voyage révolutionnaire, actes du colloque franco-néerlandais du bicentenaire de la Révolution française (Amsterdam, 12-13 octobre 1989), Hilversum, 1991, pp. 109-119. 
1790 furent célébrées plusieurs confédérations municipales et provinciales; dans les années 1791-1793, on vit à Saint-Omer des simulacres de la prise de la Bastille, de celle des Tuileries et une fête de l'Égalité; à Béthune, l'inauguration d'une statue colossale de la Liberté fut prétexte à des festivités. Ces fêtes impressionnèrent fort les réfugiés hollandais, comme l'atteste entre autres le plan réalisé par un témoin anonyme, originaire de Hollande, sur la disposition des divers corps de la garde nationale autour de l'autel et de l'arbre de la Liberté, lors de la fête de la Fédération, qui se tint le 14 juillet 1792 à Saint-Omer.

Sur la présence de patriotes hollandais à la première et grande fête révolutionnaire, la Fédération du 14 juillet 1790 sur le Champ de Mars de Paris, les informations sont légion. Parmi l'une d'entre elles, citons l'intervention du 19 juin 1790, où l'Orateur du genre humain, Anacharsis Cloots, en sa qualité de chef d'une députation de trente-trois étrangers, avait demandé à l'Assemblée nationale la permission d'assister à la Fédération : "cette solennité civique ne sera pas seulement la fête des Français, mais encore la fête du genre humain ». Contrairement aux Romains qui avaient l'habitude d'enchaîner leurs ennemis vaincus derrière leurs chars, les Français, disait Cloots, devraient inclure dans leur cortège un groupe d'étrangers qui, en France, étaient libres, alors que leur patrie demeurait dans les fers, en attendant d'être libérée par la France. Parmi les signataires de la pétition, se trouvent non seulement des célébrités comme Cloots, Price et Casanova mais aussi un groupe important de Hollandais. La pétition fit impression et fut même interrompue par des applaudissements. L'Assemblée y agréa (9). La présence de patriotes hollandais à l'événement ne fait aucun doute : malgré la pluie, ils assistèrent en nombre, aux côtés des 400000 spectateurs, à la fête des fêtes révolutionnaires, qui commémorait la chute de la Bastille et les résultats de la révolution politique qui s'était opérée jusque-là. L'écho de la fête fut si grand que, même dans la République de Guillaume V, parurent une description très précise en néerlandais et des gravures qui représentaient l'événement. Pourtant, il est impossible d'affirmer que la Fédération de 1790 ait eu une influence quelconque sur l'organisation et l'iconographie des fêtes politiques célébrées plus tard par la République batave.

Par contre, la fête de l'Unité et de l'Indivisibilité de la République, qui eut lieu à Paris le 10 août 1793 , laissa une empreinte perceptible dans quelques fêtes bataves, sur lesquelles nous allons revenir. Il est vrai qu'à Paris se trouvait un intermédiaire qui a joué un rôle non négligeable dans la diffusion de l'iconographie révolutionnaire française : H. J. Jansen, éditeur, traducteur et imprimeur hollandais s'était en effet installé à Paris et, entre

(9) J.-B. BARON Cloots (dit Anacharsis), Discours prononcé à la barre de l'Assemblée nationale, Paris, 1790. 
nombre de travaux, on lui doit une description en néerlandais de la grande fête du 10 août 1793 .

\section{La République batave et les fêtes}

La révolution batave eut lieu en janvier 1795, grâce aux troupes françaises, entre autres. Tout comme Adam et Ève, le stadhouder Guillaume V et son épouse Wilhelmina furent alors chassés du " paradis hollandais ». Au début, les Bataves voulaient surtout en finir avec le passé. Aussi peut-on observer la mise en place rapide de rites visant à annuler les mesures orangistes prises depuis 1787-1788 et à purifier l'espace public de toute allusion à la Maison d'Orange. Loranger, symbole du parti orangiste, fut alors remplacé par l'arbre de la Liberté - alias arbre de mai. Parallèlement, la révolution batave mit l'accent sur la restauration de l'autonomie municipale avec ses droits et ses privilèges. Ce qui est tout à fait différent de la tendance signalée par Mona Ozouf et de la prééminence conférée par les révolutionnaires français aux espaces ouverts, hors de la ville ou sur l'immense Champ de Mars parisien, où, durant les fêtes pouvait se réunir le peuple en masse. En France, l'enceinte, la clôture, les barrières de la ville sont interprétées comme des symboles contre-révolutionnaires, comme des atteintes à la liberté (10), tandis qu'aux Pays-Bas, elles représentent la restauration des privilèges municipaux, malmenés ou menacés par les velléités «despotiques » du stadhouder. Contrairement aux fêtes patriotes où s'exprimaient les idéaux d'un parti offensif, celles de la révolution batave figuraient les attentes d'un groupe politique dominant. Elles étaient dûment préparées et soigneusement organisées. Sous haute surveillance, le peuple n'y jouait qu'un rôle restreint. Cela vaut dès les premières fêtes de la Liberté de janvier et février 1795, célébrées un peu partout dans la République. Mais cela vaut plus encore pour les fêtes en l'honneur du traité de La Haye, qui consacrait l'alliance entre la France et la République batave. Certaines de ces fêtes officielles célébraient explicitement les alliés français, malgré le fait que l'alliance en question ait coûté à la République la gigantesque somme de cent millions de florins; d'autres, comme la fête d'Utrecht du 15 juillet 1796, voyaient participer activement les militaires français. Ces derniers marchaient dans le cortège et commémoraient les soldats français morts au combat. Deux d'entre eux libérèrent un citoyen batave prisonnier et brisèrent ses chaînes, en signe de la destruction de l'esclavage et de l'oppression. $\mathrm{Au}$ cours des premiers mois de la révolution, l'élément militaire jouait un rôle important dans ce genre de festivités et suivait en somme l'exemple de 
la France, où, à partir de l'été 1794, commençait à s'imposer dans les fêtes une symbolique militaire. L'accent posé sur les soldats français, morts au combat pour la liberté, participe de cette évolution. Dans la fête de l'alliance d'Utrecht, la fraternisation avec la France passait malgré tout au second plan; la destruction de l'ancien régime stadhouderien l'emportait de loin, ainsi qu'en témoignaient les feux de joie qui brûlaient les symboles du régime honni ou bien la libération des victimes de la tyrannie. La joie sur la restauration de ce qui avait été anéanti en 1787 par le prince d'Orange s'y mariait avec l'enthousiasme pour les acquis de la révolution batave : les Droits de l'homme proclamés fin janvier 1795, les principes de liberté, égalité et fraternité, la tolérance religieuse, les nouveaux corps politiques et juridiques, l'attente d'une reprise du commerce. Le motif principal du cortège était celui de la liberté. Comme durant l'époque des patriotes, «liberté » était le mot magique, permettant de gommer toutes les divergences entre Bataves et orangistes mais aussi entre les Bataves eux-mêmes.

La fête la plus impressionnante de la République batave fut celle qu'Amsterdam célébra le 19 juin 1795, à l'occasion de l'alliance avec la France. Les meilleurs artistes de l'époque (van der Hart, J. Andriessen, van Dreght, Bulthuis) furent invités à créer des décorations festives pour toutes les places de la ville. La plupart d'entre eux avaient de l'expérience en ce domaine, car ils avaient peint des décors de théâtre - et certains avaient conçu les décors des fêtes patriotes. Plusieurs des décorations de la fête amstellodamoise suggèrent de toute évidence une influence de la fête parisienne du 10 août 1793. Sur le Westermarkt, par exemple, se trouvait une représentation d'Hercule frappant l'hydre à sept têtes, allégorie de la victoire du peuple sur l'aristocratie et le fédéralisme des sept provinces de l'ancienne République. À y regarder de plus près, il semble bien que cette scène s'inspirait de la quatrième station de la Fête de l'Unité et de l'Indivisibilité. En cette occasion, Jacques-Louis David avait érigé sur la place des Invalides une statue colossale du Peuple français sous la figure d'Hercule, qui, de sa massue, venait d'abattre le dragon du fédéralisme tandis que, de l'autre main, il réunissait en un faisceau les 86 départements français (11). Sur une autre place, le Burgerplein (Koningsplein actuel) se trouvait une décoration représentant Hercule brisant le joug du gouvernement stadhouderien, image qui rappelle le motif d'une pièce de monnaie conçue par Dupré en 1792 (12). L'influence de la fête parisienne s'explique par le fait que le thème principal des deux fêtes était l'Unité et l'Indivisibilité. De plus, les révolutionnaires bataves aspiraient à cette date à des changements plutôt

(11) M. L. BIVER, Les fêtes révolutionnaires à Paris, Paris, 1979, pp. 70-77. L. HUNT, Politics, culture and class in the French Revolution, Berkeley, 1984, p. 87. L'auteur insiste trop sur l'opposition entre le Peuple (Hercule) et le roi.

(12) J.-C. BENZAKEN, " Hercule dans la Révolution française ou les "nouveaux travaux d'Hercule" ", in Les images de la Révolution, op. cit. pp. 203-214. 
radicaux, et Amsterdam n'était certainement pas une ville de modérés. Enfin, outre les aspirations révolutionnaires que trahissait la fête, les artistes bataves comme leurs homologues français consultaient les mêmes sources iconographiques, tel le traité d'iconologie de Ripa. Ici, sans doute, il s'agissait du manuel de Delafosse : Nouvelle iconologie.

L'Assemblée nationale, réunie à partir de 1796, discutait occasionnellement des fêtes nationales à institutionnaliser dans le cadre du premier projet de Constitution de la République. Les discussions étaient suivies de près par la revue De Democraten, rédigée par quelques Bataves unitaires, qui aspiraient donc à l'Unité et à l'indivisibilité de la République batave à venir. À l'instar de bien des contemporains, ils considéraient les fêtes nationales, calquées sur l'antique comme le moyen par excellence pour éduquer le peuple, unir les citoyens et raffermir le caractère national. Aussi la Constitution de 1798 n'omit-elle pas de réglementer l'institution des fêtes nationales et, pour ce faire, elle s'inspira de l'article 305 de la Constitution française de l'an III. L'agent (le ministre) de l'Éducation nationale en était chargé.

Curieusement, entre 1798 et 1801 , les fonctionnaires qui occupèrent ce poste manifestèrent peu d'enthousiasme à cet égard. C'est ainsi que Johannes Henricus van der Palm, agent entre 1799-1801, conférait la primauté à l'organisation de l'instruction publique et, du coup, s'intéressait peu aux fêtes nationales. Selon lui, ce genre de cérémonies ne s'accordait pas avec le caractère national et le climat hollandais. De plus, il trouvait l'exemple des fêtes françaises peu encourageant. C'est pourquoi, dans les années 1798-1801, il y eut si peu de fêtes bataves nationales. Les seules d'envergure à avoir été célébrées furent la fête en l'honneur de la Constitution du 19 mai 1798 et celle du 19 décembre 1799, qui fêtait la défaite de l'invasion anglo-russe et la victoire franco-batave. Le caractère de ces fêtes était surtout politique, contrairement à la France du Directoire, où l'accent se posait de plus en plus volontiers sur l'aspect moral et pédagogique. La République batave ignorait presque entièrement cette dimension. On y trouvait rarement des groupes d'âge ou des allégories des vertus sociales. C'est la dimension politique qui y primait (13).

Dès 1801, la République batave cherchait à réconcilier les divers partis politiques, y compris les orangistes. La fête en l'honneur de la paix d'Amiens, une des dernières festivités de l'époque, le suggère. On y trouve des allusions à la paix, au bien-être, à la prospérité et à la réconciliation. Disparaissent alors nombre de symboles bataves, tels les arbres et les statues de la Liberté. On disait qu'ils avaient servi leur but : là où règne la liberté, point besoin d'arbre ou de statue qui la symbolisent. Ainsi, les hommes poli-

(13) M. OzouF, «Symboles et fonctions des âges dans les fêtes de l'époque révolutionnaire », A.H.R.F, 1970, pp. 569-593. Et mon "purgatoire des patriotes», op. cit., pp. 117-119. 
tiques de la République batave reconnaissaient implicitement que, dans une certaine mesure, les fêtes bataves avaient été des rêves, des utopies d'une République idéale. Mais cela n'empêche pas que les fêtes célébrées après 1795 aient eu un caractère idéologique plus prononcé que celles de l'époque des patriotes.

En effet, les allusions à la tradition républicaine hollandaise et à l'ancienne iconographie politique de la République, régénérée par les patriotes s'estompèrent peu à peu, sans pour autant être remplacées par des images bataves authentiques. À l'époque batave (1795-1806), les beaux-arts n'empruntaient plus leurs motifs aux Bataves de l'Antiquité. Paradoxalement, alors même qu'ils avaient capté le nom des fondateurs de la liberté hollandaise, les révolutionnaires de 1795 délaissèrent leurs images. Entre-temps, des auteurs critiquaient le manque d'originalité des orateurs et des artistes qui contribuaient aux fêtes bataves; ils leur reprochaient de trop emprunter à la France et d'accumuler dans les décors les allégories obscures. Critiques que l'on retrouve également en France et en Allemagne où les spécialistes s'interrogeaient sur le bon usage et l'utilité de l'allégorie. Certains en venaient à conseiller de représenter certaines notions abstraites par des personnages vivants, pratique qui ne faisait l'unanimité ni en France ni en Hollande (14).

Pour conclure, il semble que la fête patriote trahit une culture orientée avant tout vers son passé national, en vue de revitaliser celui-ci, tandis que la fête batave, à la recherche d'une identité nationale pour la République nouvelle, n'y parvient qu'en empruntant cette identité pour une grande part à la France. Étant donné que les Bataves devaient tout d'abord effectuer leur révolution, dans les premières fêtes qui s'ensuivirent, c'est surtout la symbolique de la phase radicale de la Révolution française qui est invoquée. Du reste, pour ce qui est des fêtes françaises, le choix était limité. Les fédérations de 1790-1792 n'étaient plus de saison en 1795, puisque le concept politique de fédéralisme était associé désormais à la structure décentralisée de l'ancienne République, dont voulaient se débarrasser grand nombre de révolutionnaires bataves. Une fête de la Raison était impensable; jamais elle n'aurait été acceptée par des Néerlandais respectueux de la religion, tandis que la chute de Robespierre avait également discrédité la fête de l'Être suprême.

Tout cela démontre aussi qu'une réflexion de fond sur la théorie et la pratique de la Révolution française et des fêtes nationales n'était pas à l'ordre du jour en terre batave. Les fêtes bataves n'étaient pas non plus des imitations serviles des modèles parisiens, pas plus que la révolution batave ne s'était calquée sur celle de la France. Les Bataves avaient leur propre 
histoire politique et culturelle. Ils avaient déjà fait l'expérience d'un échec; ils avaient assisté de près aux mutations politiques de la Révolution française - aussi bien durant les premières années remplies d'espérances que pendant la période exceptionnelle de la Terreur. C'est avec cette double expérience et ces connaissances qu'ils entreprirent leur révolution en 1795, ce qui peut expliquer pour une part le cours original qu'elle a adopté. Dans les années qui suivirent, ils ont tenté de créer un nouvel État unitaire, d'acquérir une identité nationale où s'entremêlaient l'ancien et le nouveau, les éléments hollandais et français, tant dans le domaine de la politique que de la culture.

(traduction du néerlandais : A. Jourdan)

Frans GRIJZENHOUT Instituut Collectie Nederland De Wittenkade 86 N. L. - 1051 AK Amsterdam 